\title{
ALTERNATE CHARACTERIZATIONS OF BOUNDED VARIATION AND OF GENERAL MONOTONICITY FOR FUNCTIONS
}

\author{
BARRY BOOTON
}

\begin{abstract}
We find necessary and sufficient conditions for a function to be equal almost everywhere to a function of bounded variation. These results can be applied to broaden the class of general monotone functions.
\end{abstract}

\section{Introduction}

Being of bounded variation is a useful property for a function. A summary of many results on functions of bounded variation can be found in the monograph [1]. Many useful generalizations of the concept of bounded variation are also discussed in this survey. In addition, this work explores the impact of these generalizations on other aspects of analysis, such as rectifiable functions, the Riemann-Stieltjes integral, and Fourier series.

It is natural to attempt to generalize to $\mathbf{R}^{n}$ the original definition of a function of bounded variation on $\mathbf{R}^{1}$ or a compact subinterval due to Jordan. Brudnyi [3] describes some such approaches. In these, a multivariate function of bounded variation in some sense is an element of a broader local approximation space. In addition to summarizing results on these functions, he expands on their connection to local approximation theory.

A question that arises is whether a condition on a function can be developed in which a function retains at least some of the properties that a function of bounded variation has, but is not affected by a change in the function on a set of measure zero. The general monotone functions provide a useful setting for exploring this question. The general monotone sequences were introduced by Tikhonov in [6]; related sequences were considered earlier by Belov in [2]. Liflyand and Tikhonov extended the concept of general monotonicity to functions in [4] in multiple ways. A function $f$ defined on $(0, \infty)$ is said to be

Received July 8, 2016; received in final form January 25, 2017.

2010 Mathematics Subject Classification. 26A45, 26 A48. 
general monotone with majorant function $\beta(x) \geq 0$ if $f$ is locally of bounded variation on $(0, \infty), \lim _{x \rightarrow \infty} f(x)=0$, and there exists a constant $C>0$ such that for $x \in(0, \infty)$,

$$
\int_{x}^{2 x}|d f(t)| \leq C \beta(x) .
$$

Examples of majorant functions that they introduced included $\beta(x)=|f(x)|$ and $\beta(x)=\int_{\frac{x}{c}}^{c x}|f(t)| \frac{d t}{t}$, where $c>1$ is some constant independent of $x$. We broadly use the notation $\operatorname{GM}(\beta)$ to denote general monotone functions, and $\operatorname{GM}(c)$ to denote the special case $\beta(x)=\int_{\frac{x}{c}}^{c x}|f(t)| \frac{d t}{t}$.

In this paper, we develop necessary and sufficient conditions for a function to be equal almost everywhere to a function of bounded variation. Using these conditions, we can alter the definition of general monotone functions in a way that broadens this class of functions, but allows them to retain some key properties. We develop these conditions by considering the oscillation of a function rather than its variation. We first create some notation to express this new class of functions in a convenient manner.

Definition 1.1. For $f \in L^{1}([a, b])$, let $A f[a, b]$ denote the average of $f$ on $[a, b]$; i.e.,

$$
A f[a, b]=\frac{1}{b-a} \int_{a}^{b} f(t) d t .
$$

Definition 1.2. Let $I$ be a subinterval of $\mathbf{R}$. Let $f$ be locally integrable on $I$. Let $a \in I$. We define the functions $\lambda_{f, a}^{\prime}$ and $\lambda_{f, a}^{\prime \prime}$ on $I$ such that $\lambda_{f, a}^{\prime}(b)=$ $\lambda_{f, a}^{\prime \prime}(b)=0$ for $b \in I, b \leq a$, and for $b \in I, b>a$,

$$
\lambda_{f, a}^{\prime}(b)=\sup \sum_{k=1}^{n} \frac{1}{d_{k}-d_{k-1}} \int_{d_{k-1}}^{d_{k}}\left|f(t)-A f\left[d_{k-1}, d_{k}\right]\right| d t
$$

and

$$
\lambda_{f, a}^{\prime \prime}(b)=\sup \sum_{k=1}^{n-1}\left|A f\left[d_{k}, d_{k+1}\right]-A f\left[d_{k-1}, d_{k}\right]\right|,
$$

where the suprema are taken over all partitions $\left\{a=d_{0}<d_{1}<\cdots<d_{n}=b\right\}$ of $[a, b]$ with $n \geq 1$ and $n \geq 2$, respectively.

Note that the set of functions $\left\{f: \lambda_{f, a}^{\prime}(b)<\infty\right\}$ form a proper subspace of $B M O$. Results on the whole of $B M O$ that relate to those that follow would be of great interest.

We shall say a function $f$ measurable on $(0, \infty)$ is admissible if $f$ is locally integrable on $(0, \infty)$, and $\lim _{x \rightarrow \infty} f(x)=0$.

Definition 1.3. Let $f$ be admissible. We say that $f \in \mathrm{GM}^{\prime}(\beta)$ (or $f \in$ $\left.\operatorname{GM}^{\prime \prime}(\beta)\right)$ if there exists a constant $C>0$ and a majorant function $\beta(x) \geq 0$ 
such that for all $x \in(0, \infty)$,

$$
\begin{aligned}
\lambda_{f, x}^{\prime}(2 x) & \leq C \beta(x) \\
\left(\text { or } \lambda_{f, x}^{\prime \prime}(2 x)\right. & \leq C \beta(x)) .
\end{aligned}
$$

In the case that $\beta(x)=\int_{\frac{x}{c}}^{c x}|f(t)| \frac{d t}{t}$, we say that $f \in \mathrm{GM}^{\prime}(c)$ (or $f \in \mathrm{GM}^{\prime \prime}(c)$ ).

We shall see that $\lambda_{f, a}^{\prime}$ and $\lambda_{f, a}^{\prime \prime}$ are very closely related, and thus so are $\operatorname{GM}^{\prime}(\beta)$ and $\operatorname{GM}^{\prime \prime}(\beta)$.

In [4] and [5], Liflyand and Tikhonov described essential properties of functions $f \in \operatorname{GM}(c)$. In particular, they showed that if $f \in \operatorname{GM}(c)$, with $\int_{x}^{2 x}|d f(t)| \leq C \int_{\frac{x}{c}}^{c x}|f(t)| \frac{d t}{t}$, then for $x \in(0, \infty)$,

$$
|f(x)| \leq(C+4 \ln 2) \int_{\frac{x}{2 c}}^{\max \{2, c\} x}|f(t)| \frac{d t}{t} .
$$

Also, they showed that for such $f$, for $x, N \in(0, \infty)$, with $x<N$,

$$
\int_{x}^{N}|d f(t)| \leq 2 C(\ln c)\left(\frac{1}{\ln 2}+\frac{c}{c-1}\right) \int_{\frac{x}{c^{2}}}^{c N}|f(t)| \frac{d t}{t} .
$$

We shall refer to these conditions as Conditions 1 and 2, respectively.

We shall also see that for a function $f$ not necessarily of bounded variation on an interval $[a, b]$, but at least equal almost everywhere to such a function, then both $\lambda_{f, a}^{\prime}$ and $\lambda_{f, a}^{\prime \prime}$ can be used as a proxy for the variation of $f$. This can be applied in particular to functions in $\operatorname{GM}^{\prime \prime}(c)$. It turns out that a function $f \in \mathrm{GM}^{\prime \prime}(c)$ satisfies two conditions very similar to Conditions 1 and 2. In fact, if $f \in \mathrm{GM}^{\prime \prime}(c)$, with $\lambda_{f, x}^{\prime \prime}(2 x) \leq C \int_{\frac{x}{c}}^{c x}|f(t)| \frac{d t}{t}$, then for almost every $x \in(0, \infty)$, (1.1) holds. Likewise, for such $f$, for $x, N \in(0, \infty)$, with $x<N$,

$$
\lambda_{f, x}^{\prime \prime}(N) \leq 2 C(\ln c)\left(\frac{1}{\ln 2}+\frac{c}{c-1}\right) \int_{\frac{x}{c^{2}}}^{c N}|f(t)| \frac{d t}{t} .
$$

\section{Results}

Theorem 2.1. Let $f$ be locally integrable on $[a, b], \lambda_{f, a}^{\prime \prime}(b)<\infty$. Then

$$
\lambda_{f, a}^{\prime}(b) \leq \lambda_{f, a}^{\prime \prime}(b) .
$$

Proof. Let $\left\{a=d_{0}<d_{1}<\cdots<d_{n}=b\right\}$ be a partition of $[a, b]$. Let $\varepsilon>0$. We shall see that for $1 \leq k \leq n$, there exist $a_{k}, b_{k} \in\left(d_{k-1}, d_{k}\right)$ and $\delta_{k}>0$ such that

$$
\begin{aligned}
& \left|A f\left[b_{k}-\delta_{k}, b_{k}+\delta_{k}\right]-A f\left[a_{k}-\delta_{k}, a_{k}+\delta_{k}\right]\right| \\
& \quad>\frac{1}{d_{k}-d_{k-1}} \int_{d_{k-1}}^{d_{k}}\left|f(t)-A f\left[d_{k-1}, d_{k}\right]\right| d t-\frac{\varepsilon}{n} .
\end{aligned}
$$


For such $k$, let $g_{k}=f-A f\left[d_{k-1}, d_{k}\right]$. We can assume $\int_{d_{k-1}}^{d_{k}}\left|g_{k}(t)\right| d t>0$; otherwise, (2.1) is trivial. Let $0<\varepsilon<\frac{n}{d_{k}-d_{k-1}} \int_{d_{k-1}}^{d_{k}}\left|g_{k}(t)\right| d t$. Let $g_{k}^{+}=$ $\max \left\{0, g_{k}\right\}, g_{k}^{-}=\max \left\{0,-g_{k}\right\}$. Note that

$$
\int_{d_{k-1}}^{d_{k}} g_{k}^{+}(t) d t=\int_{d_{k-1}}^{d_{k}} g_{k}^{-}(t) d t
$$

so that

$$
\frac{1}{d_{k}-d_{k-1}} \int_{d_{k-1}}^{d_{k}} g_{k}^{+}(t) d t, \frac{1}{d_{k}-d_{k-1}} \int_{d_{k-1}}^{d_{k}} g_{k}^{-}(t) d t>\frac{\varepsilon}{2 n} .
$$

Let $a_{k}, b_{k} \in\left(d_{k-1}, d_{k}\right)$ be Lebesgue points of $g_{k}$ such that

$$
g_{k}\left(b_{k}\right) \geq \frac{1}{d_{k}-d_{k-1}} \int_{d_{k-1}}^{d_{k}} g_{k}^{+}(t) d t
$$

and

$$
g_{k}\left(a_{k}\right) \leq-\frac{1}{d_{k}-d_{k-1}} \int_{d_{k-1}}^{d_{k}} g_{k}^{-}(t) d t .
$$

Let $\delta_{k, 1}$ be such that $0<\delta_{k, 1}<\min \left\{b_{k}-d_{k-1}, d_{k}-b_{k}, \frac{\left|b_{k}-a_{k}\right|}{2}\right\}$, and for $0<$ $\delta \leq \delta_{k, 1}$,

$$
A g_{k}\left[b_{k}-\delta, b_{k}+\delta\right]>g_{k}\left(b_{k}\right)-\frac{\varepsilon}{2 n} .
$$

Likewise, let $\delta_{k, 2}$ be such that $0<\delta_{k, 2}<\min \left\{a_{k}-d_{k-1}, d_{k}-a_{k}, \frac{\left|b_{k}-a_{k}\right|}{2}\right\}$, and for $0<\delta \leq \delta_{k, 2}$,

$$
A g_{k}\left[a_{k}-\delta, a_{k}+\delta\right]<g_{k}\left(a_{k}\right)+\frac{\varepsilon}{2 n} .
$$

Let $\delta_{k}=\min \left\{\delta_{k, 1}, \delta_{k, 2}\right\}$. Then

$$
\begin{array}{rl}
\mid A & f\left[b_{k}-\delta_{k}, b_{k}+\delta_{k}\right]-A f\left[a_{k}-\delta_{k}, a_{k}+\delta_{k}\right] \mid \\
& =\left|A g_{k}\left[b_{k}-\delta_{k}, b_{k}+\delta_{k}\right]-A g_{k}\left[a_{k}-\delta_{k}, a_{k}+\delta_{k}\right]\right| \\
& >\left(g_{k}\left(b_{k}\right)-\frac{\varepsilon}{2 n}\right)-\left(g_{k}\left(a_{k}\right)+\frac{\varepsilon}{2 n}\right) \\
& \geq \frac{1}{d_{k}-d_{k-1}} \int_{d_{k-1}}^{d_{k}}\left|f(t)-A f\left[d_{k-1}, d_{k}\right]\right| d t-\frac{\varepsilon}{n} .
\end{array}
$$

Therefore, letting $r_{k}=\min \left\{a_{k}, b_{k}\right\}, s_{k}=\max \left\{a_{k}, b_{k}\right\}$,

$$
\begin{aligned}
& \sum_{k=1}^{n} \frac{1}{d_{k}-d_{k-1}} \int_{d_{k-1}}^{d_{k}}\left|f(t)-A f\left[d_{k-1}, d_{k}\right]\right| d t \\
& \quad<\sum_{k=1}^{n}\left(\left|A f\left[s_{k}-\delta_{k}, s_{k}+\delta_{k}\right]-A f\left[r_{k}-\delta_{k}, r_{k}+\delta_{k}\right]\right|+\frac{\varepsilon}{n}\right)
\end{aligned}
$$




$$
\begin{aligned}
\leq & \sum_{k=1}^{n}\left(\left|A f\left[r_{k}-\delta_{k}, r_{k}+\delta_{k}\right]-A f\left[d_{k-1}, r_{k}-\delta_{k}\right]\right|\right. \\
& +\left|A f\left[r_{k}+\delta_{k}, s_{k}-\delta_{k}\right]-A f\left[r_{k}-\delta_{k}, r_{k}+\delta_{k}\right]\right| \\
& +\left|A f\left[s_{k}-\delta_{k}, s_{k}+\delta_{k}\right]-A f\left[r_{k}+\delta_{k}, s_{k}-\delta_{k}\right]\right| \\
& \left.+\left|A f\left[s_{k}+\delta_{k}, d_{k}\right]-A f\left[s_{k}-\delta_{k}, s_{k}+\delta_{k}\right]\right|\right)+\varepsilon \leq \lambda_{f, a}^{\prime \prime}(b)+\varepsilon .
\end{aligned}
$$

Since $\varepsilon>0$ was arbitrary,

$$
\sum_{k=1}^{n} \frac{1}{d_{k}-d_{k-1}} \int_{d_{k-1}}^{d_{k}}\left|f(t)-A f\left[d_{k-1}, d_{k}\right]\right| d t \leq \lambda_{f, a}^{\prime \prime}(b) .
$$

Since $\left\{a=d_{0}<d_{1}<\cdots<d_{n}=b\right\}$ was an arbitrary partition of $[a, b]$,

$$
\lambda_{f, a}^{\prime}(b) \leq \lambda_{f, a}^{\prime \prime}(b) .
$$

Corollary 2.2. $\operatorname{GM}^{\prime \prime}(\beta) \subset \operatorname{GM}^{\prime}(\beta)$.

TheORem 2.3. Let $f$ be locally integrable on $[a, b], \lambda_{f, a}^{\prime}(b)<\infty$. Then

$$
\lambda_{f, a}^{\prime \prime}(b) \leq \frac{9}{2} \lambda_{f, a}^{\prime}(b) .
$$

Proof. Let $\left\{a=d_{0}<d_{1}<\cdots<d_{n}=b\right\}$ be a partition of $[a, b]$ with $n \geq 2$. Let $\delta=\min _{1 \leq k \leq n}\left(d_{k}-d_{k-1}\right)$. For $1 \leq k \leq n$, let $s_{k}$ be such that $2^{s_{k}} \delta \leq$ $d_{k}-d_{k-1}<2^{s_{k}+1} \delta$. For such $k$, for $0 \leq j \leq 2^{s_{k}}$, let

$$
d_{k, j}=d_{k-1}+\frac{j}{2^{s_{k}}}\left(d_{k}-d_{k-1}\right)
$$

so that for $1 \leq j \leq 2^{s_{k}}$,

$$
\delta \leq d_{k, j}-d_{k, j-1}<2 \delta
$$

By relabelling, let

$$
\left\{a=c_{0}<c_{1}<\cdots<c_{m}=b\right\}=\bigcup_{k=1}^{n} \bigcup_{j=0}^{2^{s_{k}}}\left\{d_{k, j}\right\} .
$$

Of course, this means that for $1 \leq l \leq m, \delta \leq c_{l}-c_{l-1}<2 \delta$. We shall see that for $1 \leq l \leq m-1$,

$$
\left|A f\left[c_{l}, c_{l+1}\right]-A f\left[c_{l-1}, c_{l}\right]\right| \leq \frac{9}{2} \frac{1}{c_{l+1}-c_{l-1}} \int_{c_{l-1}}^{c_{l+1}}\left|f(t)-A f\left[c_{l-1}, c_{l+1}\right]\right| d t .
$$

Let $p_{l}=\frac{c_{l}-c_{l-1}}{c_{l+1}-c_{l-1}}$. We have $\frac{1}{3}<p_{l}<\frac{2}{3}$. Also, note that

$$
\begin{aligned}
A f\left[c_{l-1}, c_{l+1}\right] & =\frac{1}{c_{l+1}-c_{l-1}}\left(\int_{c_{l-1}}^{c_{l}} f(t) d t+\int_{c_{l}}^{c_{l+1}} f(t) d t\right) \\
& =p_{l} A f\left[c_{l-1}, c_{l}\right]+\left(1-p_{l}\right) A f\left[c_{l}, c_{l+1}\right] .
\end{aligned}
$$


Thus,

$$
\begin{aligned}
& \frac{1}{c_{l+1}-c_{l-1}} \int_{c_{l-1}}^{c_{l}}\left(f(t)-A f\left[c_{l-1}, c_{l+1}\right]\right) d t \\
& \quad=p_{l}\left(A f\left[c_{l-1}, c_{l}\right]-A f\left[c_{l-1}, c_{l+1}\right]\right)=p_{l}\left(1-p_{l}\right)\left(A f\left[c_{l-1}, c_{l}\right]-A f\left[c_{l}, c_{l+1}\right]\right) .
\end{aligned}
$$

Likewise,

$$
\begin{gathered}
\frac{1}{c_{l+1}-c_{l-1}} \int_{c_{l}}^{c_{l+1}}\left(f(t)-A f\left[c_{l-1}, c_{l+1}\right]\right) d t \\
=\left(1-p_{l}\right)\left(A f\left[c_{l}, c_{l+1}\right]-A f\left[c_{l-1}, c_{l+1}\right]\right) \\
=p_{l}\left(1-p_{l}\right)\left(A f\left[c_{l}, c_{l+1}\right]-A f\left[c_{l-1}, c_{l}\right]\right) .
\end{gathered}
$$

Therefore,

$$
\begin{aligned}
& \frac{1}{c_{l+1}-c_{l-1}} \int_{c_{l-1}}^{c_{l+1}}\left|f(t)-A f\left[c_{l-1}, c_{l+1}\right]\right| d t \\
& \geq \frac{1}{c_{l+1}-c_{l-1}}\left(\left|\int_{c_{l-1}}^{c_{l}}\left(f(t)-A f\left[c_{l-1}, c_{l+1}\right]\right) d t\right|\right. \\
& \left.\quad+\left|\int_{c_{l}}^{c_{l+1}}\left(f(t)-A f\left[c_{l-1}, c_{l+1}\right]\right) d t\right|\right) \\
& =2 p_{l}\left(1-p_{l}\right)\left|A f\left[c_{l}, c_{l+1}\right]-A f\left[c_{l-1}, c_{l}\right]\right| \geq \frac{4}{9}\left|A f\left[c_{l}, c_{l+1}\right]-A f\left[c_{l-1}, c_{l}\right]\right| .
\end{aligned}
$$

We estimate

$$
\sum_{k=1}^{n-1}\left|A f\left[d_{k}, d_{k+1}\right]-A f\left[d_{k-1}, d_{k}\right]\right| .
$$

For $1 \leq k \leq n$, since

$$
A f\left[d_{k-1}, d_{k}\right]=\frac{1}{2^{s_{k}}} \sum_{j=1}^{2^{s_{k}}} A f\left[d_{k, j-1}, d_{k, j}\right]
$$

there exists $j_{k}, 1 \leq j_{k} \leq 2^{s_{k}}-1$, such that

$$
\begin{aligned}
& \left|A f\left[d_{k, j_{k}}, d_{k, j_{k}+1}\right]-A f\left[d_{k, j_{k}-1}, d_{k, j_{k}}\right]\right| \\
& \quad=\left|A f\left[d_{k, j_{k}}, d_{k, j_{k}+1}\right]-A f\left[d_{k-1}, d_{k}\right]\right|+\left|A f\left[d_{k-1}, d_{k}\right]-A f\left[d_{k, j_{k}-1}, d_{k, j_{k}}\right]\right| .
\end{aligned}
$$

Let

$$
D=\left\{l: 1 \leq l \leq m-1, \text { and } c_{l}=d_{k, j_{k}} \text { for some } k, 1 \leq k \leq n\right\}
$$

Then

$$
\begin{aligned}
& \sum_{l=1}^{m-1}\left|A f\left[c_{l}, c_{l+1}\right]-A f\left[c_{l-1}, c_{l}\right]\right| \\
& \quad=\sum_{1 \leq l \leq m-1, l \notin D}\left|A f\left[c_{l}, c_{l+1}\right]-A f\left[c_{l-1}, c_{l}\right]\right|
\end{aligned}
$$




$$
\begin{aligned}
& +\sum_{k=1}^{n}\left|A f\left[d_{k, j_{k}}, d_{k, j_{k}+1}\right]-A f\left[d_{k, j_{k}-1}, d_{k, j_{k}}\right]\right| \\
= & \sum_{1 \leq l \leq m-1, l \notin D}\left|A f\left[c_{l}, c_{l+1}\right]-A f\left[c_{l-1}, c_{l}\right]\right| \\
& +\sum_{k=1}^{n}\left(\left|A f\left[d_{k, j_{k}}, d_{k, j_{k}+1}\right]-A f\left[d_{k-1}, d_{k}\right]\right|\right. \\
& \left.+\left|A f\left[d_{k-1}, d_{k}\right]-A f\left[d_{k, j_{k}-1}, d_{k, j_{k}}\right]\right|\right) \\
\geq & \sum_{k=1}^{n-1}\left|A f\left[d_{k}, d_{k+1}\right]-A f\left[d_{k-1}, d_{k}\right]\right| .
\end{aligned}
$$

Thus,

$$
\begin{aligned}
& \sum_{k=1}^{n-1}\left|A f\left[d_{k}, d_{k+1}\right]-A f\left[d_{k-1}, d_{k}\right]\right| \\
& \leq \sum_{1 \leq l \leq m-1, l \text { odd }}\left|A f\left[c_{l}, c_{l+1}\right]-A f\left[c_{l-1}, c_{l}\right]\right| \\
& \quad+\sum_{1 \leq l \leq m-1, l \text { even }}\left|A f\left[c_{l}, c_{l+1}\right]-A f\left[c_{l-1}, c_{l}\right]\right| \\
& \quad \leq \frac{1}{4}\left(\sum_{1 \leq l \leq m-1, l \text { odd }} \frac{1}{c_{l+1}-c_{l-1}} \int_{c_{l-1}}^{c_{l+1}}\left|f(t)-A f\left[c_{l-1}, c_{l+1}\right]\right| d t\right. \\
& \left.\quad+\sum_{1 \leq l \leq m-1, l \text { even }}^{c_{l+1}}\left|f(t)-A f\left[c_{l-1}, c_{l+1}\right]\right| d t\right) \\
& \leq
\end{aligned}
$$

Since $\left\{a=d_{0}<d_{1}<\cdots<d_{n}=b\right\}$ was an arbitrary partition of $[a, b]$ with $n \geq 2$,

$$
\lambda_{f, a}^{\prime \prime}(b) \leq \frac{9}{2} \lambda_{f, a}^{\prime}(b)
$$

Corollary 2.4. $\operatorname{GM}^{\prime}(\beta) \subset \operatorname{GM}^{\prime \prime}(\beta)$.

TheOREM 2.5. Let $f$ be of bounded variation on $[a, b]$. Then

$$
\lambda_{f, a}^{\prime \prime}(b) \leq \int_{a}^{b}|d f(t)| .
$$

Proof. Let $\left\{a=d_{0}<d_{1}<\cdots<d_{n}=b\right\}$ be a partition of $[a, b]$ with $n \geq 2$. Let

and let

$$
A=\left\{k: A f\left[d_{k-1}, d_{k}\right] \leq A f\left[d_{k}, d_{k+1}\right]\right\}
$$

$$
B=\left\{k: A f\left[d_{k-1}, d_{k}\right]>A f\left[d_{k}, d_{k+1}\right]\right\} .
$$


For $1 \leq k \leq n$, let $d_{k, 1}, d_{k, 2} \in\left(d_{k-1}, d_{k}\right)$ be such that

$$
f\left(d_{k, 1}\right) \leq A f\left[d_{k-1}, d_{k}\right] \leq f\left(d_{k, 2}\right) .
$$

In the case $1 \in A$, we construct inductively a finite sequence $\left\{k_{i}\right\}_{i=0}^{m}$. Let $k_{0}=1$. For $i \geq 0$ even, if $k \in A$ for $k_{i} \leq k \leq n-1$, let $m=i+1$ and $k_{m}=n$, and the sequence is complete. Otherwise, let $k_{i+1}=\min \left\{k>k_{i}: k \in B\right\}$. Likewise, for $i \geq 1$ odd, if $k \in B$ for $k_{i} \leq k \leq n-1$, let $m=i+1$ and $k_{m}=n$, and the sequence is complete. Otherwise, let $k_{i+1}=\min \left\{k>k_{i}: k \in A\right\}$. Then

$$
\begin{aligned}
& \sum_{k=1}^{n-1}\left|A f\left[d_{k}, d_{k+1}\right]-A f\left[d_{k-1}, d_{k}\right]\right| \\
& =\sum_{1 \leq i \leq m, i \text { odd }}\left(A f\left[d_{k_{i}-1}, d_{k_{i}}\right]-A f\left[d_{k_{i-1}-1}, d_{k_{i-1}}\right]\right) \\
& \quad+\sum_{1 \leq i \leq m, i \text { even }}\left(A f\left[d_{k_{i-1}-1}, d_{k_{i-1}}\right]-A f\left[d_{k_{i}-1}, d_{k_{i}}\right]\right) \\
& \quad \leq \sum_{1 \leq i \leq m, i \text { odd }}\left|f\left(d_{k_{i}, 2}\right)-f\left(d_{k_{i-1}, 1}\right)\right|+\sum_{1 \leq i \leq m, i \text { even }}\left|f\left(d_{k_{i}, 1}\right)-f\left(d_{k_{i-1}, 2}\right)\right| \\
& \quad \leq \int_{a}^{b}|d f(t)| .
\end{aligned}
$$

A similar proof yields an identical result in the case $1 \in B$.

Since $\left\{a=d_{0}<d_{1}<\cdots<d_{n}=b\right\}$ was an arbitrary partition of $[a, b]$ with $n \geq 2$,

$$
\lambda_{f, a}^{\prime \prime}(b) \leq \int_{a}^{b}|d f(t)| .
$$

Corollary 2.6. $\operatorname{GM}(\beta) \subset \operatorname{GM}^{\prime \prime}(\beta)$.

THEOREM 2.7. Let $f$ be locally integrable on $[a, b], \lambda_{f, a}^{\prime \prime}(b)<\infty$. Then there exists $g$ of bounded variation on $[a, b]$ such that $f=g$ a.e., and

$$
\int_{a}^{b}|d g(t)| \leq \lambda_{f, a}^{\prime \prime}(b) \text {. }
$$

Proof. We define $g$ on $[a, b]$ such that

$$
\begin{aligned}
g(a) & =\frac{1}{2}\left(\liminf _{h \rightarrow 0^{+}} \frac{1}{h} \int_{a}^{a+h} f(t) d t+\limsup _{h \rightarrow 0^{+}} \frac{1}{h} \int_{a}^{a+h} f(t) d t\right), \\
g(b) & =\frac{1}{2}\left(\liminf _{h \rightarrow 0^{+}} \frac{1}{h} \int_{b-h}^{b} f(t) d t+\limsup _{h \rightarrow 0^{+}} \frac{1}{h} \int_{b-h}^{b} f(t) d t\right)
\end{aligned}
$$

and for $x \in(a, b)$,

$$
g(x)=\frac{1}{2}\left(\liminf _{h \rightarrow 0^{+}} \frac{1}{2 h} \int_{x-h}^{x+h} f(t) d t+\limsup _{h \rightarrow 0^{+}} \frac{1}{2 h} \int_{x-h}^{x+h} f(t) d t\right) .
$$


If $x \in(a, b)$ is a Lebesgue point of $f$, then

$$
g(x)=\lim _{h \rightarrow 0^{+}} \frac{1}{2 h} \int_{x-h}^{x+h} f(t) d t=f(x)
$$

and thus $g=f$ a.e.

Next we see that $g$ is of bounded variation on $[a, b]$, and

$$
\int_{a}^{b}|d g(t)| \leq \lambda_{f, a}^{\prime \prime}(b)
$$

Let $\left\{a=d_{0}<d_{1}<\cdots<d_{n}=b\right\}$ be a partition of $[a, b]$. Let $\varepsilon>0$. Let $1 \leq k \leq n-1$. If $d_{k}$ is a Lebesgue point of $f$, then there exists $h_{k}$ such that

$$
0<h_{k}<\min \left\{\frac{d_{k}-d_{k-1}}{2}, \frac{d_{k+1}-d_{k}}{2}\right\}
$$

and

$$
g\left(d_{k}\right)-\frac{\varepsilon}{2 n}<\frac{1}{2 h_{k}} \int_{d_{k}-h_{k}}^{d_{k}+h_{k}} f(t) d t<g\left(d_{k}\right)+\frac{\varepsilon}{2 n} .
$$

Otherwise, let

$$
\tau=\limsup _{h \rightarrow 0^{+}} \frac{1}{2 h} \int_{d_{k}-h}^{d_{k}+h} f(t) d t-\liminf _{h \rightarrow 0^{+}} \frac{1}{2 h} \int_{d_{k}-h}^{d_{k}+h} f(t) d t>0 .
$$

Then there exist $h_{k, 1}, h_{k, 2}$ such that

$$
0<h_{k, 1}, h_{k, 2}<\min \left\{\frac{d_{k}-d_{k-1}}{2}, \frac{d_{k+1}-d_{k}}{2}\right\}
$$

and

$$
\begin{aligned}
\frac{1}{2 h_{k, 1}} \int_{d_{k}-h_{k, 1}}^{d_{k}+h_{k, 1}} f(t) d t & <\liminf _{h \rightarrow 0^{+}} \frac{1}{2 h} \int_{d_{k}-h}^{d_{k}+h} f(t) d t+\frac{\tau}{2} \\
& =\limsup _{h \rightarrow 0^{+}} \frac{1}{2 h} \int_{d_{k}-h}^{d_{k}+h} f(t) d t-\frac{\tau}{2} \\
& <\frac{1}{2 h_{k, 2}} \int_{d_{k}-h_{k, 2}}^{d_{k}+h_{k, 2}} f(t) d t
\end{aligned}
$$

Since $F_{d_{k}}(h)=\frac{1}{2 h} \int_{d_{k}-h}^{d_{k}+h} f(t) d t$ is continuous for $0<h<\min \left\{d_{k}-d_{k-1}\right.$, $\left.d_{k+1}-d_{k}\right\}$, by the Intermediate Value theorem, there exists $h_{k}, \min \left\{h_{k, 1}\right.$, 
$\left.h_{k, 2}\right\}<h_{k}<\max \left\{h_{k, 1}, h_{k, 2}\right\}$, such that

$$
\begin{aligned}
& \frac{1}{2 h_{k}} \int_{d_{k}-h_{k}}^{d_{k}+h_{k}} f(t) d t \\
& \quad=\frac{1}{2}\left(\liminf _{h \rightarrow 0^{+}} \frac{1}{2 h} \int_{d_{k}-h}^{d_{k}+h} f(t) d t+\limsup _{h \rightarrow 0^{+}} \frac{1}{2 h} \int_{d_{k}-h}^{d_{k}+h} f(t) d t\right)=g\left(d_{k}\right) .
\end{aligned}
$$

Similarly, there exist $h_{0}, h_{n}$ such that $0<h_{0}<\frac{d_{1}-d_{0}}{2}, 0<h_{n}<\frac{d_{n}-d_{n-1}}{2}$,

$$
g\left(d_{0}\right)-\frac{\varepsilon}{2 n}<\frac{1}{h_{0}} \int_{d_{0}}^{d_{0}+h_{0}} f(t) d t<g\left(d_{0}\right)+\frac{\varepsilon}{2 n}
$$

and

$$
g\left(d_{n}\right)-\frac{\varepsilon}{2 n}<\frac{1}{h_{n}} \int_{d_{n}-h_{n}}^{d_{n}} f(t) d t<g\left(d_{n}\right)+\frac{\varepsilon}{2 n} .
$$

Then

$$
\begin{aligned}
\sum_{k=1}^{n} \mid & g\left(d_{k}\right)-g\left(d_{k-1}\right) \mid \\
< & \left|\frac{1}{2 h_{1}} \int_{d_{1}-h_{1}}^{d_{1}+h_{1}} f(t) d t-\frac{1}{h_{0}} \int_{d_{0}}^{d_{0}+h_{0}} f(t) d t\right| \\
& +\sum_{k=2}^{n-1}\left|\frac{1}{2 h_{k}} \int_{d_{k}-h_{k}}^{d_{k}+h_{k}} f(t) d t-\frac{1}{2 h_{k-1}} \int_{d_{k-1}-h_{k-1}}^{d_{k-1}+h_{k-1}} f(t) d t\right| \\
& +\left|\frac{1}{h_{n}} \int_{d_{n}-h_{n}}^{d_{n}} f(t) d t-\frac{1}{2 h_{n-1}} \int_{d_{n-1}-h_{n-1}}^{d_{n-1}+h_{n-1}} f(t) d t\right|+\varepsilon \\
\leq & \left|A f\left[d_{0}+h_{0}, d_{1}-h_{1}\right]-A f\left[d_{0}, d_{0}+h_{0}\right]\right| \\
& +\sum_{k=1}^{n-1}\left(\left|A f\left[d_{k}-h_{k}, d_{k}+h_{k}\right]-A f\left[d_{k-1}+h_{k-1}, d_{k}-h_{k}\right]\right|\right. \\
& \left.+\left|A f\left[d_{k}+h_{k}, d_{k+1}-h_{k+1}\right]-A f\left[d_{k}-h_{k}, d_{k}+h_{k}\right]\right|\right) \\
& +\left|A f\left[d_{n}-h_{n}, d_{n}\right]-A f\left[d_{n-1}+h_{n-1}, d_{n}-h_{n}\right]\right|+\varepsilon \\
\leq & \lambda_{f, a}^{\prime \prime}(b)+\varepsilon .
\end{aligned}
$$

Since $\varepsilon>0$ was arbitrary,

$$
\sum_{k=1}^{n}\left|g\left(d_{k}\right)-g\left(d_{k-1}\right)\right| \leq \lambda_{f, a}^{\prime \prime}(b) .
$$

Since $\left\{a=d_{0}<d_{1}<\cdots<d_{n}=b\right\}$ was an arbitrary partition of $[a, b]$,

$$
\int_{a}^{b}|d g(t)| \leq \lambda_{f, a}^{\prime \prime}(b) \text {. }
$$


Corollary 2.8. Let $f \in \operatorname{GM}^{\prime \prime}(\beta)$. Then there exists $g \in \operatorname{GM}(\beta)$ such that $f=g$ a.e.

Corollary 2.9. Let $f \in \operatorname{GM}^{\prime \prime}(c)$, with $\lambda_{f, x}^{\prime \prime}(2 x) \leq C \int_{\frac{x}{c}}^{c x}|f(t)| \frac{d t}{t}$. Then for almost every $x \in(0, \infty)$, (1.1) holds.

Corollary 2.10. If $f \in \mathrm{GM}^{\prime \prime}(c)$, with $\lambda_{f, x}^{\prime \prime}(2 x) \leq C \int_{\frac{x}{c}}^{c x}|f(t)| \frac{d t}{t}$, then for $x, N \in(0, \infty)$, with $x<N,(1.2)$ holds.

Proof. Since there exists $g \in \operatorname{GM}(c)$ such that $f=g$ a.e, using Theorem 2.5,

$$
\begin{aligned}
\lambda_{f, x}^{\prime \prime}(N) & =\lambda_{g, x}^{\prime \prime}(N) \leq \int_{x}^{N}|d g(t)| \\
& \leq 2 C(\ln c)\left(\frac{1}{\ln 2}+\frac{c}{c-1}\right) \int_{\frac{x}{c^{2}}}^{c N}|g(t)| \frac{d t}{t} \\
& =2 C(\ln c)\left(\frac{1}{\ln 2}+\frac{c}{c-1}\right) \int_{\frac{x}{c^{2}}}^{c N}|f(t)| \frac{d t}{t} .
\end{aligned}
$$

\section{REFERENCES}

[1] J. Appell, J. Banaś and N. Merentes, Bounded variation and around, DeGruyter Studies in Nonlinear Analysis and Applications, de Gruyter, Berlin, 2014. MR 3156940

[2] A. S. Belov, Remarks on mean convergence (boundedness) of partial sums of trigonometric series, Mat. Zametki 71 (2002), no. 6, 807-817. English translation in: Math. Notes 71 (2002), nos. 5-6, 739-748. MR 1933102

[3] Y. Brudnyi, Multivariate functions of bounded $(k, p)$-variation, Banach spaces and their applications in analysis (Oxford, OH, 2006), de Gruyter, Berlin, 2007, pp. 3757. MR 2374699

[4] E. Liflyand and S. Tikhonov, The Fourier transforms of general monotone functions, Analysis and mathematical physics, Trends in Mathematics, Birkhäuser, Basel, 2009, pp. 377-395. MR 2724621

[5] E. Liflyand and S. Tikhonov, A concept of general monotonicity and applications, Math. Nachr. 284 (2011), no. 8-9, 1083-1098. MR 2839773

[6] S. Tikhonov, Trigonometric series with general monotone coefficients, J. Math. Anal. Appl. 326 (2007), no. 1, 721-735. MR 2277815

Barry Booton, Department of Mathematical Sciences, Florida Atlantic University, 777 Glades Road, Boca Raton, FL 33431, USA

E-mail address: bbooton@fau.edu 